\title{
Normal and Pathological Reactions to Severe Stress: Their Features and Management
}

\author{
DA Alexander
}

\begin{abstract}
It is unethical and incompetent to deny the scale and nature of human reactions to trauma. Those who provide care for victims of trauma should be familiar with normal and pathological reactions following trauma and know how to identify those at high risk of developing adverse reactions and problems of adjustment.

Methods of treating and preventing post-traumatic conditions have not been evaluated with sufficient rigour. There are, however, some guiding principles to ensure a rational approach to the management of victims of trauma.
\end{abstract}

I can sympathise with everything, except suffering. I cannot sympathise with that. It is too ugly, too horrible, too distressing... the less said about life's sores the better.

(The Picture of Dorian Gray, Oscar Wilde, 1856-1900)

\section{Introduction}

Man is no stranger to adversity. However, even the worst examples of human suffering and tragedy have not always been met with concern and compassion. Victims have been blamed for their own misfortune, rejected (as though their suffering were infectious), or held in suspicion on the grounds that their complaints were inspired by some ulterior motive such as the quest for compensation(1). Even the survivors of the holocaust were not always believed when they returned with their stories of almost ineffable degradation, pain and suffering. Most strikingly, in their medical notes there was often no mention of their period of incarceration in concentration camps.

It would be too simplistic to dismiss such views and attitudes as merely the product of malice or ignorance. Underlying such attitudes (which still prevail) there is a more subtle principle operating, viz, that of the need to shield ourselves from the suffering of others and to enable us to hold on to the assumption that we would never have to endure such suffering. If we regard these victims as weak, stupid, careless or exploitative we can try to reassure ourselves that we are not likely to share their fate.
Whilst self-protective, this persistent reluctance throughout history to acknowledge the suffering of victims of trauma is to inflict upon them a 'second injury'.

\section{The military position}

The chronicles of military combat have provided a potentially rich vein of knowledge about the impact of trauma. Unfortunately, despite there being such evidence dating back at least to the GraecoRoman wars, the lessons often painfully learned were conveniently forgotten between military campaigns.

Those medical personnel who had to deal with the horrors of military combat may have had their ability to understand the suffering of combatants compromised by two other influences. First, prior to effective chemical anaesthesia and analgesia, military surgeons were fully exposed to the unadulterated suffering and distress of the victims of combat. Perhaps one way to cope with this was to distance themselves psychologically from this suffering and to take refuge in some form of denial, eg, only the weak and unmotivated complain. Second, military surgeons were also cast in a difficult dilemma. To whom do they owe their allegiance - to the wounded and suffering combatant or to the state?

In these enlightened times it is easy to dismiss the conduct and attitudes of military medical personnel during the earlier wars as callous, indifferent and unprofessional. If we are really to understand the impact of severe stress, then we must try to understand their behaviour and attitudes as well. There are many opportunities to do so in the military texts and in the poetry of, for example, Wilfred Owen (1893-1918).

\section{A diagnostic hydra?}

Military medical history reveals a proliferation of diagnostic terms, each fashioned in an attempt to make sense of acute stress reactions in combatants. In the 17 th century 'Nostalgia' was coined by Hofer to describe the melancholic longing for home, and impaired sleep and appetite observed in Swiss and Napoleonic troops. The American War of Independence sired 'soldier's heart', 'irritable heart' and the
E-mail:

mental.health.FH.@abdn.ac.uk 
eponymous da Costa's syndrome. 'Shellshock' emerged as the explanation during the Great War to account for what we now regard as combat-related psychopathology. The scale of this psychopathology is reflected in the fact that about 200,000 men were rendered unfit for further combat duties because of it.

What characterized the early attempts to understand acute reactions to stress was the quest for physical explanations; an attempt no doubt consistent with the pre-eminence of the physical rather than the psychological sciences. Diarrhoea, cigarette smoking, fever, overly tight accoutrements, carbon dioxide poisoning, and micro-haemorrhagic damage to the cortex caused by exploding artillery shells were among the favourites.

There were implications of this emphasis on physical aetiologies. The first was that politicians and military command were largely relieved of any responsibility for the emotional suffering of combatants. To have acknowledged publicly the psychological horror of combat would have compromised the steady flow of new recruits. Second, treatments were themselves commonly physical and commonly involved the use of electric shocks and even subcutaneous injections of ether. So vigorous were some of these treatments that there were fatalities.

The competing aetiological view that 'shell shocked' soldiers were suffering from some form of 'moral inferiority' was an equally comfortable one for the authorities. Those so afflicted could be dealt with through dishonourable discharge or even death at the hands of the firing squad.

\section{Post-traumatic Stress Disorder}

The introduction of this disorder into the medical nomenclature is a further demonstration of the influence of social and political forces in the shaping of medical history.

During the Vietnam War the level of psychopathology in the combat zone was relatively low but, on demobilization, veterans displayed a high level of psychiatric pathology and problems of social adjustment. Concerned by this unexpected circumstance the US Government commissioned the American Psychiatric Association to address this issue. Their deliberations led to the introduction of Post-traumatic Stress Disorder into the Third Edition of the Diagnostic and Statistical Manual (DSM-III, 1980). Subsequent revisions were brought out in 1987 (DSM - III -R) and in 1994 (DSM IV). In 1992 the International Classification of Disease and Related Health Problems (ICD- 10, WHO) also accommodated this diagnosis.

Although these two taxonomies differ to some extent in relation to their diagnostic criteria, the common elements are that : (a) the victim needs to have been exposed to an exceptionally stressful event; (b) the victim needs to suffer repeated intrusive memories, nightmares or 'flashbacks' associated with the trauma; (c) the victim must display avoidance of reminders of the trauma (eg, going back to the scene of the event or talking about it), and (d) the victim experiences hyperarousal (as displayed in an exaggerated acoustic startle response). Hyperarousal is often accompanied by hypervigilance, ie, a heightened sense of risk and vulnerability.

The diagnosis of PTSD has been overused, and its diagnostic criteria have been challenged(2). On the other hand, there have been five major gains following its introduction.

(i) By focussing on the primary aetiological role of the stressor (and not on some putative moral or personality defect of the victim) the suffering of many individuals has been legitimized.

(ii) It extended the concept of trauma beyond military combat to include civilian disasters and more commonplace but serious incidents such as road traffic accidents and assaults.

(iii) It stimulated more open debate about reactions to trauma.

(iv) It encouraged research on a number of fronts, including biochemistry, neuroimaging, psychiatry and sociology.

(v) It finally introduced some order into the otherwise chaotic proliferation of diagnostic labels.

\section{Epidemiology}

PTSD constitutes a genuine health problem. Community surveys(3) suggest that the lifetime risk of PTSD is about one per cent; a figure which is comparable to that for schizophrenia. Estimates vary because of different methods of assessment but the following figures(4) give some idea of its prevalence following different kinds of trauma: rape (35\%), burns (45\%), RTAs $(10 \%)$, terrorist attacks $(18 \%)$, and Falkland War (22\%). It should also be noted that PTSD commonly occurs in association with other conditions including depression, anxiety and substance abuse.

\section{Additional post-traumatic conditions}

In addition to PTSD the ICD-10 recognizes other important reactions as consequences of trauma.

(i) Acute stress reaction

Patients will appear to be in a state of shock and display a constricted field of consciousness with impaired concentration and orientation. The clinical picture is likely to fluctuate and involve depression, agitation, anxiety, social withdrawal and autonomic over- 
reactivity. It usually abates within a couple of days after the victim is removed from the stressful situation.

(ii) Panic attack

Such attacks are associated with sudden severe anxiety of unpredictable onset. They are characterized by various symptoms such as palpitations, profuse sweating, an inexplicable sense of impending doom, tremor, dyspnoea, chest pain, paraesthesiae and a choking sensation. Hyperventilation is common, and may lead to the hyperventilation syndrome.

These attacks may endure for several hours but most commonly they are short-lived, peaking within about 10 minutes.

\section{(iii) Dissociation}

This is a disorder of acute onset and may manifest itself in amnesia, stupor, disturbed physical function (eg, paralysis or sensory loss). Recovery is usually sudden and complete within a few days or months following the trauma.

\section{Normal reactions to severe stress}

Psychiatric diagnoses should not be overused. With the rare exception of a posttraumatic psychotic condition, normal and pathological reactions are on a continuum and are usually distinguished by:

- the intensity of the reactions

- the duration of the reactions, and

- the extent to which they render the victim dysfunctional

The following reactions are common after stress.

- Numbness and denial

These are nature's way of shielding us against otherwise overwhelming events.

- Fear

Fear is a natural biological response to threat and triggers the fight/flight response.

\section{- Depression}

Most trauma include some kind of loss, and loss commonly triggers low mood (see also Grief reactions below).

- Elation

This is not common but it has been reported particularly in combat troops ('combat rush'). It may be associated with a raised pain threshold perhaps due to the effect of natural endorphins.

- Anger

Once victims realise what has happened anger is a common reaction. A legitimate target may not present itself thus those around the victims (including rescuers, family, colleagues and caregivers) may become the target of this anger.

- Irritability

This is not a dramatic reaction but it can seriously compromise even the most stable work and family relationships.

- Guilt

Victims may suffer 'survivor guilt' particularly if others have died in the same trauma. (This guilt may contribute to the increased rate of suicide after trauma.)

- Impaired sleep

Autonomic hyperarousal, flashbacks and nightmares commonly disturb sleep. Nightmares can be so intense that they deter victims from even trying to sleep. Alterations to the sleep architecture include increased REM sleep and decreased deep sleep.

- Cognitive changes

The victims' recollection of the trauma is very often of events happening as though they were in slow motion. Also, they may display 'tunnel vision' such that they focus on the main source of threat to the detriment of their awareness of more peripheral details. These reactions account in part for the distorted recall displayed by victims after trauma.

- Helplessness

This is almost a hallmark of trauma - a sense of acute vulnerability and of feeling unable to alter the outcome of events.

NB: Because PTSD is not diagnosed until several weeks after the trauma, its definitive features of flashbacks, avoidant behaviour and hyperarousal can be viewed as 'normal' in the shorter term.

\section{Grief reactions}

Trauma nearly always entails loss. Although the loss of a loved one is perhaps the most obvious source of grief, there are other significant sources including the loss of a limb through amputation (surgical or traumatic), loss of looks (through burns or other forms of disfigurement) and the loss of property and possessions (commonly seen among refugees). Following major catastrophe and military combat, there may be multiple losses, inducing a cumulative effect.

Acute grief reactions may overlap with some of the symptoms of post-traumatic stress, but one should be alert also to the following.

- Shock, denial and disbelief

At this stage victims may be unable to accept the reality of the loss. One needs therefore to be patient and to keep offering the same information until they are able to take it in.

- Apathy

They may display a complete lack of drive and initiative even to the point of being unable to cater for their own basic needs.

- Pining and searching

This is evidenced by what appear to the onlooker to be fruitless attempts to find evidence that the dead are still alive. 
- Acute generalised distress

This commonly occurs in waves or 'pangs'. There may be alternating episodes of coping and of being overwhelmed by grief.

- Agitation and irritability

Although explicit anger may be seen, these reactions are more common. The bereaved commonly show a reduced threshold for what they see to be the inadequacies of others, even those who seek to help.

\section{Prognosis}

There is no single trauma which will lead to a post-traumatic syndrome in all those exposed to it. There are however some factors which relate to a poor prognosis and have implications for subsequent management.

(a) Trauma-related factors

- Prolonged exposure (eg, being trapped, held hostage).

- Threat to life ('threat' as perceived by the victim even if it does not entail objective risk).

- Multiple deaths and/or mutilation.

- Sudden and unexpected (ie, there is no time to prepare).

- Man made(eg, assault and combat rather than natural events such as floods and earthquakes).

(b) Victim-related factors

- Serious personal injury. (What seems 'serious' to the patient will in part depend on the personal meaning of the injury and may not correspond to what is clinically defined as 'serious'.)

- Previous psychiatric history (including alcohol and substance abuse).

- Particularly severe acute stress reaction.

- A profound sense of helpless and/or hopelessness.

- Where the trauma has a special meaning to the victim. (The death or injury to a child nearly always entails a 'special meaning'.)

- A particularly anxious personality.

- A lack of social and family support.

- Previous trauma to which the victim has not yet adjusted (including an earlier bereavement).

- Concurrent life stresses. (It is particularly important to be aware of these as they may compromise the victim's ability to cope with the recent trauma.)

Principles of management following severe stress Too soon following trauma, counselling (and other forms of early intervention such as debriefing) may not be helpful; indeed, they may do more harm by prematurely reexposing the victim to the traumatic event.
However, prior to the stage whereby counselling and other interventions may become legitimate options, we can help individuals by means of what has been called by Raphael 'psychological first aid'(5).

Below is an abbreviated version of her principles.

- Comfort and protect

Because victims of trauma are often stunned by what has happened, physical comfort (including personal touch, clothing and food) can be particularly reassuring. It is also important to ensure that the victims do not inflict further harm on themselves. (RTA victims have been known to wander blindly in front of on-coming traffic.)

- Counteract helplessness

Victims commonly report how 'useless' (and even guilty) they felt at the scene of a major incident. Thus, it can be helpful to encourage them, if they are physically able, to help at the scene by, for example, comforting the injured, stopping oncoming traffic, alerting the emergency services etc.

- Re-establish a sense of order and control A trauma induces a sense of chaos and personal vulnerability, if only fleetingly. These are extremely unpleasant experiences and can lead to panic in some. A good antidote is to convey to the victims that somebody is in charge and that the incident is now under control.

- Expression of feelings

In the early stages after trauma it is unlikely to be helpful to do more than to allow individuals to express feelings which are close to the surface. Very basic physical needs (eg, those for safety and comfort) are more likely to take priority. Prematurely provoking individuals into 'sharing their feelings' may merely retraumatise them and make them feel worse.

If guilt is expressed avoid superficial and unjustified reassurance. Unless your information is watertight you may find subsequently that their guilt is indeed unjustified!

- Provision of accurate information.

Because victims of trauma feel stunned and numbed or in a state of disbelief you may find that they can only take in a little information at a time. Be prepared to 'titrate' the dose and to repeat it regularly. Again, beware of speculation or of giving false information. If you are not sure (eg, about the safety and welfare of the victim's friends or loved ones), it is safer to admit uncertainty whilst reassuring the victim that you will make every effort to answer any questions as soon as you can.

- Psychological triage

After trauma, using the prognostic indicators above, it is helpful to identify 
those who may be at particular risk of subsequent psychopathology and/or problems of adjustment. At this stage it can also be helpful to give those at risk clear information as to where help may be obtained later.

The management of acute grief reactions

It should be remembered that most individuals negotiate a bereavement without professional help, and, therefore, it is important not to 'pathologize' normal reactions. There are however steps we can take to help the bereaved in the early stages.

- Listening

When in doubt, say nothing. It is often better to be present and offer physical comfort rather than 'talking at' the bereaved.

- Clichés and platitudes

Silence is certainly preferable to these, for example, 'you're lucky to be alive' and 'at least you have another child at home'. (Both of these were said to a young mother who had driven down a dual carriageway in the wrong direction, colliding with an on-coming car and witnessed her young daughter being catapulted from the family car and killed by a lorry.)

- Normalize

Reassure victims that their reactions are normal. Commonly they feel they are being 'stupid', 'weak' or are losing their minds.

- Expression of feelings

We can gently facilitate the expression of underlying feelings and crying. It is not good practice to do more than this. There is no need to go 'mining' for deep-rooted, underlying feelings. (Bear in mind, at the earliest stages after a loss, victims are often too stunned to appreciate fully what has happened.)

\section{- Toleration}

The bereaved can be irritable and intolerant of others, including those who are seeking to help. Such reactions can commonly be seen in the treatment room where patients complain about 'careless' debridement and venepuncture(6). It is best not to take personal offence and become defensive.

Formal treatment of post-traumatic syndromes Unfortunately, despite the prevalence of such syndromes, the number of carefully conducted randomized trials is small, and findings vary because of different research designs and outcome measures(7). Thus, at best the following are only guidelines.

(i) Medication

\section{- Benzodiazepines}

These may be used in the case of acute overwhelming symptoms, particularly if these symptoms jeopardize medical care. However they should only be used for a short period and with caution for patients with head injury and a history of benzodiazepine dependence. They have little effect on core PTSD symptoms.

- Antidepressants

Tricyclic antidepressants and MAOIs may be used to alleviate intrusive symptoms (but dietary caution is required in the case of the latter group). The new SSRIs (eg, fluoxetine) may also help intrusive and avoidant symptoms although drug-induced insomnia may be a problem.

- Anticonvulsants

Carbamazepine may alleviate insomnia and intrusive phenomena, and valproate may reduce hyperarousal and avoidant /numbing symptoms.

- Beta blockers

Propranol may reduce autonomic hyperarousal (it usually has no effect, however, on anxious thoughts).

- Neuroleptics

These are not regarded as frontline treatments unless there is clear evidence of psychosis or of overwhelming aggression and/or self-destructive tendencies.

(ii) Psychotherapy

Most effective psychological treatments involve:

- exposing patients to the original trauma either in vivo or in imagination (using Graded Exposure or Systematic Desensitization), and

- helping patients to revise their thoughts about the trauma (Cognitive Behavioural Therapy has considerable empirical support).

More recently, there has been enthusiastic support and increasing empirical evidence for the value of Eye Movement Desensitization and Reprocessing Therapy, but its theoretical basis has yet to be established(8). Clinical experience also suggests that Group Therapy (including self help groups) may be of help to victims and to their families.

\section{Debriefing}

Despite good training, careful staff selection and the 'inoculation effect' of previous experience, it is now widely acknowledged that providing care for trauma victims can have a major impact on $\operatorname{staff}(9,10,11)$.

Although psychological debriefing following trauma has been used for many years, particularly by the military, the term 'debriefing' itself only became fashionable after the efforts of, for example, Mitchell(12) and Dyregrov(13). It should be noted that debriefing was never intended as a 'therapy'; it was intended for use with normal individuals who had encountered an abnormal event. It was also intended to be used in a group with a trained debriefer. 
Although different authorities espouse different models of debriefing, the essential features are as follows.

- Normalization of emotional and other psychological reactions.

- Enabling the natural expression of emotions.

- Identifying successes in terms of how the incident had been managed.

- Identifying problems (rather than focusing on 'failures') and solutions.

- Enabling staff to 'let go' of the incident in order that they can go home or move on to other duties.

Although it was intended as a psychoprophylaxis, recent empirical evidence(7) has failed to confirm its early promise. Moreover, some findings suggest that debriefing may make individuals worse. The fact that debriefing might have a 'psychonoxious' effect is not at all surprising because any therapeutic intervention (surgical, pharmacological or psychiatric) has the potential to do harm as well as good. The key issue is do we know best when to use it, by whom it should be used and for whom it should be used? Good answers to these questions are awaited. However, we can probably now assert that mandatory debriefing should be avoided as it assumes that all potential members of a debriefing group are equally ready emotionally to undergo an experience which has the potential to re-traumatize, through re-exposure to the initial trauma.

Despite the empirical evidence, and despite the obvious risk of re-traumatizing individuals, there is a widespread enthusiasm among organizations for debriefing, and, generally, participants report personal satisfaction after having undergone debriefing. It may be, therefore, that we should now be looking towards 'practice-based evidence' rather than 'evidence-based practice'.

\section{Conclusions}

(i) Human reactions to extreme stress are complex, but there is considerable consistency across incidents and experiences.

(ii) Historically, there has been a reluctance to acknowledge human suffering after trauma, but this can no longer be justified. (iii) Post-traumatic Stress Disorder and other post-traumatic syndromes are not the hallmarks of those who are weakwilled, naïve, or bent on financial gain. (Such symptoms do not generally remit following the resolution of compensation claims.)

(iv) We should not 'pathologize' normal reactions to trauma by subjecting every victim, willing or otherwise, to treatment'. It is now possible to identify 'high risk' individuals and circumstances in order that interventions (psychological and psychopharmacotherapeutic) may be delivered selectively.

(v) Effective trauma care requires attention to physical and psychological phenomena among the primary victims and among those who care for them.

\section{References}

1. Alexander, DA. Trauma research: a new era. Fournal of Psychosomatic Research. (1996). 41, 1-5.

2. Breslau, N. and Davis GC. Posttraumatic Stress Disorder. The Stressor Criterion. Fournal of Nervous and Mental Disease. (1987). 175, 255-264.

3. Helzer JE, Robins LN. and McEvoy L. Posttraumatic stress disorder in the general population: Findings of the Epidemiologic Catchment Area survey. New England fournal of Medicine (1987). 317, 1630-1634.

4. Alexander DA. Psychological aspects of trauma. In: Key Topics in Trauma (Eds, I, Greaves, K, Porter, D, Burke). Bios Scientific Publishers: Oxford. (1997).

5. Raphael B. When Disaster Strikes. Hutchinson: New York. (1986).

6. Alexander, D.A. Burn victims after a major disaster: reactions of patients and their care-givers. Burns. (1993). 19, 105-109.

7. O'Brien LS. Traumatic Events and Mental Health. Cambridge University Press: Cambridge. (1998).

8. Shapiro F. Eye movement desensitization and reprocessing: Basic principles, protocols and procedures. Guildford Press: New York(1995).

9. Alexander DA. and Atcheson SF. Psychiatric aspects of trauma care: a survey of nurses and doctors. Psychiatric Bulletin. (1998). 22, 132-136.

10. Figley CR. Compassion Fatigue. Coping with Secondary Traumatic Stress Disorder in those who treat the Traumatized. Brunner/Mazel: New York. (1995).

11. Alexander DA. and Klein S. (in press). Ambulance personnel: the impact of accident and emergency work on mental health and emotional well-being. British Journal of Psychiatry.

12. Mitchell JT. When disaster strikes... The critical incident stress debriefing process. Fournal of Emergency Medical Services (1983). 8, 36-39.

13. Dyregrov A. Caring for helpers in a disaster situation: psychological debriefing. Disaster Management. (1989). 2, 25-30. 